\title{
MAIN-SEQUENCE STARS AND THE STAR FORMATION HISTORY OF THE OUTER DISK IN THE LARGE MAGELLANIC CLOUD ${ }^{1}$
}

\author{
J. S. Gallagher, ${ }^{2}$ J. R. Mould, ${ }^{3}$ E. de FeiJter, ${ }^{2,4}$ J. Holtzman, ${ }^{5}$ B. Stappers, ${ }^{3}$ A. Watson, ${ }^{5}$ \\ J. Trauger, ${ }^{6}$ G. E. Ballester, ${ }^{7}$ C. J. Burrows, ${ }^{8}$ S. Casertano, ${ }^{9}$ J. T. Clarke, ${ }^{7}$ D. Crisp, ${ }^{6}$ \\ R. E. Griffiths, ${ }^{8}$ J. J. Hester, ${ }^{10}$ J. Hoessel, ${ }^{2}$ J. Krist, ${ }^{8}$ L. D. MATthews, ${ }^{11}$ \\ P. A. SCOWEn, ${ }^{10}$ K. R. StAPElfeld, ${ }^{6}$ AND J. A. WestPhal ${ }^{12}$ \\ Received 1995 September 28; accepted 1996 February 29
}

\begin{abstract}
Using the Wide Field Planetary Camera 2 on the Hubble Space Telescope, we have obtained a deep color-magnitude diagram in $V$ - and $I$-band equivalents for more than 2000 stars in a patch of the outer disk of the Large Magellanic Cloud (LMC). Aperture photometry is feasible from these data with good signal-to-noise ratio for stars with $V \leq 25$, which allows us for the first time to construct a colormagnitude diagram for LMC disk stars on the lower main sequence, extending beyond the oldest mainsequence turnoff point. We analyze the structure of the main-sequence band and overall morphology of the color-magnitude diagram to obtain a star formation history for the region. A comparison between the distribution of stars across the main-sequence band for $M_{V} \leq 4$ and a stellar population model constrains historical star formation rates within the past $3 \mathrm{Gyr}$. The stellar populations in this region sample the outer LMC disk for stars with ages of $1 \mathrm{Gyr}$ or older that have had time to spatially mix. The structure of the main-sequence band requires that star formation occurred at a roughly constant rate during most of the past $\approx 3 \mathrm{Gyr}$. However, the distribution of subgiant stars indicate that a pronounced peak in the star formation rate likely occurred about $2 \mathrm{Gyr}$ ago, prior to which the star formation rate had not been enhanced for several Gyr. Studies over timescales of more than $3 \mathrm{Gyr}$ require a separation of the effects of star formation history and the chemical evolution on the LMC color-magnitude diagrams, which is difficult to achieve without additional constraints. If lower main-sequence stars in the LMC have moderate metallicities, then the age for most LMC disk stars is less than about 8 Gyr.
\end{abstract}

Subject headings: galaxies: photometry - galaxies: stellar content - Hertzsprung-Russell diagram Magellanic Clouds - stars: evolution

\section{INTRODUCTION}

As the nearest galaxy, the Large Magellanic Cloud (LMC) is an invaluable laboratory for testing ideas about the structure and evolution of galaxies (see, e.g., Herschel 1847; Hodge 1989; Westerlund 1990). One of the most fundamental questions to be explored is the star formation history of this galaxy. How has the LMC formed stars over its $\approx 14$ Gyr lifetime? Our current perspective on this question is largely based on studies of star clusters, which can be dated from their colors and stellar luminosity functions (see,

\footnotetext{
${ }^{1}$ Based on observations with the NASA/ESA Hubble Space Telescope, obtained at the Space Telescope Science Institute, which is operated by AURA, Inc. under NASA contract 5-26555.

2 Department of Astronomy, 5534 Sterling, University of Wisconsin, 475 North Charter Street, Madison, WI 53706-1582.

${ }^{3}$ Mount Stromlo and Siding Springs Observatories, Australian National University, Weston Creek Post Office, ACT 2611, Australia.

${ }^{4}$ University of Utrecht, Utrecht, Netherlands.

${ }^{5}$ Lowell Observatory, Mars Hill Road, Flagstaff, AZ 86001; and Department of Astronomy, New Mexico State University, Box 30001, Department 4500, Las Cruces, NM 88003-8001.

${ }^{6}$ Jet Propulsion Laboratory, 4800 Oak Drive, Pasadena, CA 91109.

${ }^{7}$ Department of Atmospheric and Oceanic Sciences, University of Michigan, 2455 Hayward, Ann Arbor, MI 48109.

${ }^{8}$ Space Telescope Science Institute, 3700 San Martin Drive, Baltimore, MD 21218.

${ }^{9}$ Department of Physics and Astronomy, Johns Hopkins University, 3400 North Charles Street, Baltimore, MD 21218.

${ }_{10}$ Department of Physics and Astronomy, Box 871504, Arizona State University, Tempe, AZ 85287-1504.

${ }^{11}$ Astronomy Program, Department of Earth and Space Science, SUNY at Stony Brook, Stony Brook, NY 11794-2100.

12 Division of Geological and Planetary Sciences, California Institute of Technology, Pasadena, CA 91125.
}

e.g., Hodge 1987; van den Bergh 1991). LMC star clusters for which color-magnitude diagrams have been measured show a bimodal distribution with most clusters having ages of $<4 \mathrm{Gyr}$, in combination with a few true globular clusters that are 12-15 Gyr old (Jensen, Mould, \& Reid 1988; Da Costa 1991; van den Bergh 1991).

This age distribution implies that the production of rich, compact star clusters in the LMC has been highly episodic. However, dense star cluster formation efficiencies may depend on the mode of star formation as well as the overall star formation rate (SFR) (O'Connell, Gallagher, \& Hunter 1994). The majority of LMC stars are unlikely to have formed in dense star clusters. Thus, it is not clear that the evolution of dense star clusters is the same as the evolution of the LMC as a whole. Furthermore, Hodge (1988) finds that the birthrate of smaller LMC star clusters, while also locally episodic, in some regions might have had a comparatively smooth global history over the past 10 Gyr. Determinations of star formation histories from the field star components of the LMC are essential for a proper description of its evolutionary history.

Progress in this area has been hampered by the limited ability of ground-based telescopes to obtain accurate optical photometry of faint stars in the crowded LMC. At low brightness levels, blending between stars and confusion with background galaxies are problems, even in the relatively low density outer disk of the LMC. Furthermore, lower mass stars are red and thus have spectral energy distributions that peak at wavelengths beyond $0.7 \mu \mathrm{m}$, where terrestrial airglow is bright. Ground-based photometry therefore rapidly loses accuracy for LMC stars fainter than 
about $V=22$ and cannot readily reach below the oldest main-sequence turnoff (see, e.g., Hardy et al. 1984; Stryker 1984; Bertelli et al. 1992).

These difficulties can be greatly reduced by obtaining stellar photometry with the Hubble Space Telescope (HST). The $H S T$ provides an image quality of $\leq 0$ " 1 , works against a dark sky in the near-infrared, and allows excellent stargalaxy separation. In this paper we present and interpret deep photometry obtained with Wide Field and Planetary Camera 2 (WFPC2) on the HST in filters that are close to the $V$ and $I$ bands. The observations were made for an LMC outer disk star field located at $\alpha_{2000}=05^{\mathrm{h}} 14^{\mathrm{m}} 44^{\mathrm{s}} .2$, $\delta_{2000}=-65^{\circ} 17^{\prime} 43^{\prime \prime}$, about $12^{\prime}(180 \mathrm{pc})$ from the intermediate-age LMC star cluster NGC 1866. This field has no evidence for ongoing star formation, appears to be relatively uniform, and has sufficiently low surface density that we would be able to avoid crowding even at faint levels with the WFPC2. Such an undistinguished location is a good place to explore the average star formation history of the LMC disk.

In $\S 2$ of this paper we summarize previous observations of LMC outer disk field star color-magnitude diagrams. We present our WFPC 2 data in $\S 3$, analyze these results in $\S 4$, and present a description of the LMC disk star formation history in $\S 5$. A derivation of the LMC lower main sequence stellar luminosity function from these data will be given by Holtzman et al. (1996).

\section{FLUCTUATING STAR FORMATION RATES IN THE LARGE MAGELLANIC CLOUD}

The populations of long-lived, low-mass field stars in galaxies record the histories of star formation. Unfortunately, low-mass main-sequence stars are difficult to observe beyond the Milky Way (see, e.g., Mighell \& Butcher 1992; Smecker-Hane et al. 1994). Observations of field stars have the advantage of relative insensitivity to the locations of star formation, since most star clusters in the LMC dissolve after 1 Gyr (Hodge 1988), and stars within the disk will spatially mix over comparable timescales. A disadvantage of field stellar populations is their range in ages and metallicities which complicates the astrophysical interpretation of measured properties.

Ground-based photographic surveys of LMC field stars by Butcher (1977), Frogel \& Blanco (1983), Hardy et al. (1984), and Stryker $(1983,1984)$, among others, show a prevalent intermediate-age stellar population. This point is especially clear in Stryker's (1984) comparison of an LMC outer disk field star color-magnitude diagram with that of the nearby, old LMC globular star cluster NGC 2257, where she finds the field stellar population has half the age and higher metallicity than NGC 2257.

Bertelli et al. (1992) used deep, high-quality $B V$ CCD observations to photometer field stars in and around three intermediate-age LMC star clusters, including NGC 1866. The resulting stellar densities on the color-magnitude Hess diagrams were averaged over selected regions and matched to predictions of theoretical stellar evolution models. Their preferred model has a major star formation event extending over most of the past $4 \mathrm{Gyr}$ and producing about $80 \%$ of all disk stars. The remaining older stars are born at a lower rate during the preceding 5-12 Gyr. More recently, Westerlund, Linde, \& Lyngå (1995) and Vallenari et al. (1996a, b) have reached similar conclusions for several other LMC fields.
Our current model for the star formation history of the LMC thus consists of an initial star-forming event at about 10-14 Gyr that produced a few globular star clusters and an old, metal-poor disk component (see Suntzeff et al. 1992), followed by several Gyr of inactivity that ended about 4 Gyr ago when the main disk of the LMC was produced (Feast 1995).

Such a remarkable star formation pattern is important to understand because it differs from present-day, mildly fluctuating SFRs observed in typical IBm galaxies (see, e.g., Gallagher, Hunter, \& Tutukov 1984) and may be related to the large numbers of very blue galaxies seen at moderate redshifts of $z=0.3-1$ associated with look-back times of ₹3-8 Gyr (see, e.g., Broadhurst, Ellis, \& Shanks 1988; Cowie, Songaila, \& Hu 1991; Lilly 1993; Koo et al. 1995). We therefore focus our investigation on the LMC disk star formation history during the past several Gyr with particular emphasis on the role of starbursts.

\section{WFPC2 OBSERVATIONS AND RESULTS}

The WFPC2 and its on-orbit performance have been described by Burrows (1995) and Trauger et al. (1994). In 1994 May we obtained 4000 s exposures in both the F555W and F814W filters, which are similar to the $V$ and $I$ bands, respectively. The images were processed in a standard way to remove bias, correct for dark current and hot pixels, and apply flat fields (Holtzman et al. 1995b). These images have an unusual low-level ripple pattern that was not removed by standard processing. This slightly decreases our sensitivity at faint levels but does not substantially affect the brighter stars that we discuss in this paper. Each of the three WFPC2 wide field camera CCDs covers slightly less than $80 \times 80 \mathrm{arcsec}^{2}$, with a resolution of $0.1 \mathrm{pixel}^{-1}$. At our adopted LMC distance of $52 \mathrm{kpc}, 1^{\prime \prime}$ corresponds to $0.25 \mathrm{pc}$, and our photometry is for an area of $\approx 1200 \mathrm{pc}^{2}$.

The combined images were corrected for cosmic-ray events and were digitally photometered. Stars are point sources, which allows us to discriminate against the inclusion of most background galaxies among the brighter stars studied here. This was done by selecting only objects that appeared to be unresolved for photometry. Aperture photometry was carried out at Wisconsin and at Lowell with 3 and 2 pixel radii apertures, respectively, on the three Wide Field Camera images. Because these images were obtained when WFPC2 was operating at its colder setting of $-88^{\circ} \mathrm{C}$ and had significant sky background, we did not need to apply a linear ramp to correct for CCD charge transfer efficiency. Our star identification routines found more than 2000 stars in our four WFPC2 images. An example of one of the WFPC2 images is shown in Figure 1.

Random photometric errors were calculated from the CCD read noise and count statistics within the stellar images and their local backgrounds. The importance of measurement errors can be judged from data on stars with $V \approx 25$, where we find $\sigma_{V} \approx \sigma_{555 \mathrm{~W}}=0.06$ and $\sigma_{V-I} \approx$ $\sigma_{555 \mathrm{~W}-814 \mathrm{~W}}=0.09$. An external check on these data is provided by the resulting color-magnitude diagram (CMD; see Fig. 2). An upper bound on $\sigma_{V-I}$ can be found by assuming that all of the color spread in the main sequence at $V=25$ is due to photometric errors. The observed color full width at half-maximum star density is $\Delta(V-I)_{\mathrm{fwhm}}=0.25 \mathrm{mag}$, implying that $\sigma_{V-I} \leq 0.11 \mathrm{mag}$. This comparison shows that the internal error estimates are reasonable and that the LMC main sequence has an intrinsically narrow spread in 


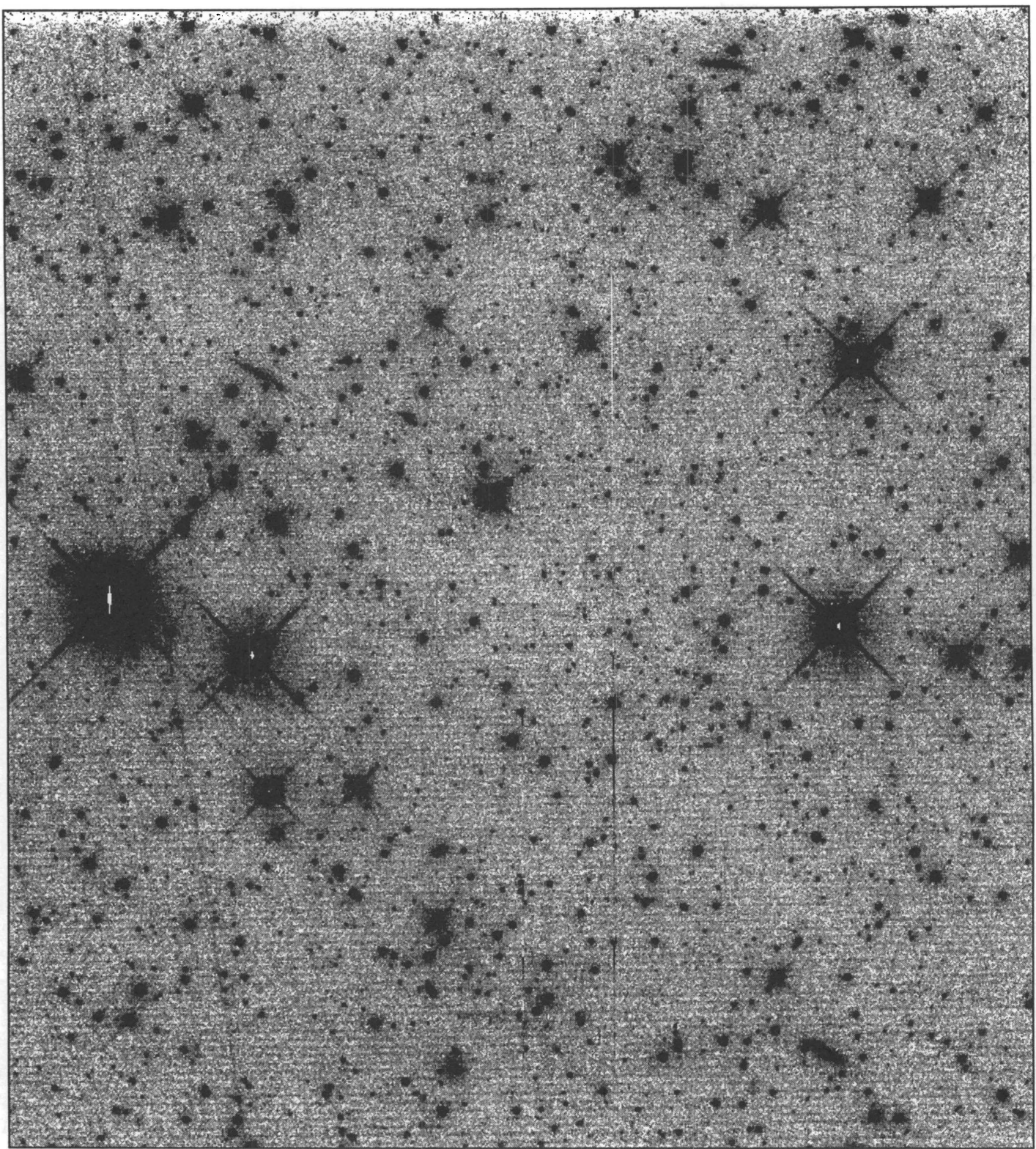

FIG. 1.-Patch of the LMC outer disk as observed by the WFPC2 Wide Field Camera No. 2 CCD. It illustrates the low star density of the region (which allows accurate aperture photometry), the presence of background galaxies seen through the LMC disk, and bias ripple in these data. This image was made from four F814W WFPC2 exposures for a total exposure time of $4000 \mathrm{~s}$. The faintest stars have $I \approx 26$.

$V-I$ color. We also note the presence of more widely scattered points in the CMD than would be predicted by the above estimates of $\sigma_{V-I}$. These are generally lower weight measurements where confusion, a cosmic ray, or some other problem has reduced the quality of the photometry.

We also explored the more difficult issue of systematic errors in the photometry. Both the Lowell and Wisconsin photometry were corrected to the standard 5 pixel Wide Field Camera apertures of Holtzman et al. (1995a) using stars with no nearby neighbors. The aperture correction was assumed to be field independent. Based on point-spread function models, we estimate that this simplification introduces an additional $1 \%-2 \%$ scatter in the final data set. We then intercompared the final Lowell and Wisconsin instrumental magnitudes and found agreement to within errors. The Lowell photometry was adopted because it used the smaller aperture and thus has better signal-to-noise ratios for fainter stars. A further comparison was made between
CCDs using instrumental colors as a fiducial, and we found that the zero points agree to within 0.03 mag between the three Wide Field CCDs. Thus for $V \leq 23.5$, we conservatively adopt $\sigma_{V}=0.03$ and $\sigma_{V-I}=0.05$ as the limiting precision of our instrumental magnitudes.

We transformed the WFPC2 magnitudes to the Cousins $V-I$ color system using the equations of Holtzman et al. (1995a), which have estimated errors of $\leq 0.05 \mathrm{mag}$, including the possibility of systematic offsets in zero points. However, we emphasize that this is a preliminary calibration that may not exactly apply to the moderatemetallicity stars that we are observing in the LMC. Our observed color-magnitude diagram is presented in Figure 2, where we show only data with $V \leq 26$, corresponding to stars with $M_{V}<7.2$ for a true LMC distance modulus of $(m-M)_{0}=18.6\left(D_{\text {LMC }}=52 \mathrm{kpc} ;\right.$ Crotts, Kunkel, \& Heathcote 1995) with an extinction $A_{V}=0.2$ corresponding to $E(V-I)=0.10$. Our observations reach deeper than

\section{(C) American Astronomical Society - Provided by the NASA Astrophysics Data System}




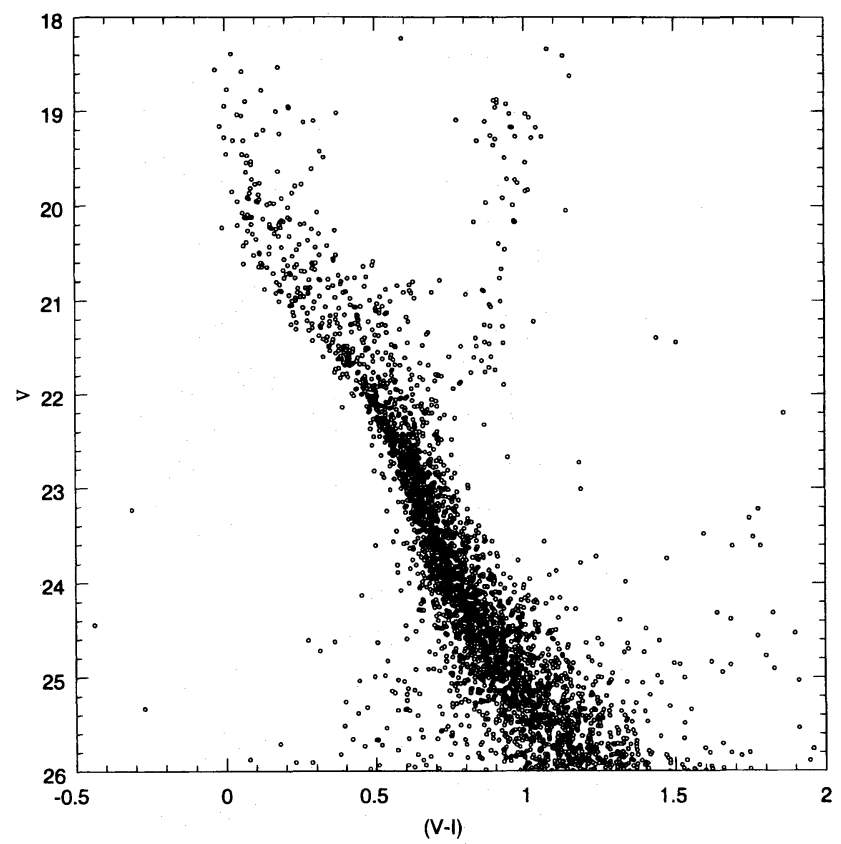

Fig. 2.-This color-magnitude diagram was derived from 2 pixel $(0$ ".2) radius aperture photometry carried out at Lowell Observatory. In this investigation we are emphasizing the part of the CMD with $V \leq 25$. The data have been transformed to the Kron-Cousins $V-I$ system from the WFPC2 F555W and F814W filters.

$V=26$, and the full data set and results from profile-fitting photometry will be discussed in a later paper (Holtzman et al. 1996). Stars with $V \leq 25$ are preferred for studies of the CMD because they have internal photometric errors of $\leq 0.1$ mag in their $V-I$ colors.

Figure 2 has several interesting features that can be compared with the ground-based observations of an LMC field near NGC 1866 by Bertelli et al. (1992). The blue edge of the main-sequence band is sharper than the red edge. The stellar density is also higher near the blue edge. This effect is most pronounced for stars with $25>V>22$, where the main sequence shows a concentration of stars along the blue side of the main-sequence band. At brighter magnitudes, the main-sequence band appears to be more uniformly populated, and at fainter limits, a variety of effects compress the main-sequence band to the point where photometric errors and metallicity spread become factors. For $V<20$, the statistics are poor because of the small size of our field, and thus the properties of the main-sequence band and upper red giant branch are not well determined from these data.

A well-defined subgiant branch is associated with the faintest main-sequence turnoff near $V=22.5$, and a second turnoff is visible at $V \approx 21.5$. The red side of the mainsequence band is ragged for stars with $20<V<23$, as expected if numerous main-sequence turnoffs exist but are individually obscured by the low numbers of stars. The presence of at least two distinct main-sequence turnoffs indicates that some episodic star formation has occurred in this region (see Smecker-Hane et al. 1996).

\section{COMPARISONS WITH MODEL STELLAR POPULATIONS}

\subsection{Simple Fits to Isochrones}

A convenient starting point for modeling the stellar populations in our LMC field can be obtained by compar- ing our data with CMD models made by combining the Yale isochrones (Green, Demarque, \& King 1987). While these are not the most modern isochrone models, they offer the advantage of wide age and metallicity coverage. We therefore used these models to obtain a qualitative understanding of possible evolutionary histories.

We constructed simulated CMDs for a range of possible star formation histories. The models are based on the $Z=0.01, Y=0.3$ Yale isochrones and contain 1770 stars. Two of these models are illustrated in Figure 3. These plots show results for a constant SFR model and for a model with a base constant SFR and a factor of 3 boost in SFR 2 Gyr ago. We find the best qualitative agreement with our observations for models that have a relatively flat SFR over the past 3 Gyr but experienced a significant SFR enhancement about 2 Gyr ago. Using these models, we make a first estimate that the lower luminosity turnoff near $M_{V} \approx 3.5$ occurs at an age of about $8 \pm 3 \mathrm{Gyr}$, where the precise age depends on the stellar models and metallicities. The second main-sequence turnoff is $1 \mathrm{mag}$ brighter, and is made up of stars with an age of $\approx 2 \mathrm{Gyr}$, corresponding to part of the high SFR interval in the life of the LMC in the standard LMC evolutionary model (Feast 1995). We now turn to a new quantitative technique to learn more about the star formation history of the LMC disk within the last few Gyr.

\subsection{Main-Sequence Band-Fitting Technique}

Since the bulk of a low-mass star's nuclear-burning lifetime is spent in the main-sequence evolutionary phase, this region of the H-R diagram normally contains most of the nuclear-burning stars within each mass interval. For example, in a galaxy with constant SFR, main-sequence stars of each mass will spread in temperature and luminosity to make the main-sequence band. The zero-age main sequence (ZAMS) produces a sharply defined blue edge to this band, while the rapid redward evolution following core exhaustion of hydrogen produces a red edge. Within this band, the number of stars distributed along a cut at constant luminosity depends on the relationship between stellar lifetime and color (see below). By comparing the observed relative numbers of stars across the main-sequence band at a given luminosity to model predictions derived from theoretical stellar evolution model tracks, we can recover information about historical variations in SFRs.

This approach to measuring galactic star formation histories has the advantage of improved statistics over most other methods; e.g., there are approximately 10 times as many $\approx 1.5 M_{\odot}$ stars in the main-sequence band of a constant SFR galaxy as in all of their post-main-sequence, nuclear-burning evolution stages combined. Furthermore, in looking along lines of constant luminosity, we compare stars with very similar masses but with various ages, so a detailed knowledge of the form of the initial mass function (IMF) is not required to derive star formation histories with this technique (see, e.g., Eggleton, Fitchett, \& Tout 1989).

In the main-sequence luminosity range $1<M_{V}<4$, the central concentration of nuclear energy generation drives evolving stars relatively rapidly to the red (Iben \& Rood 1970; Renzini \& Fusi-Pecci 1988). However, even in this region, stars spend the majority of their main-sequence lives close to the ZAMS. Thus, for a constant SFR model, most main-sequence stars in this luminosity interval should always be on the blue side of the band. Stars with $M_{V} \geq 4$ evolve more homologously during core hydrogen burning, 


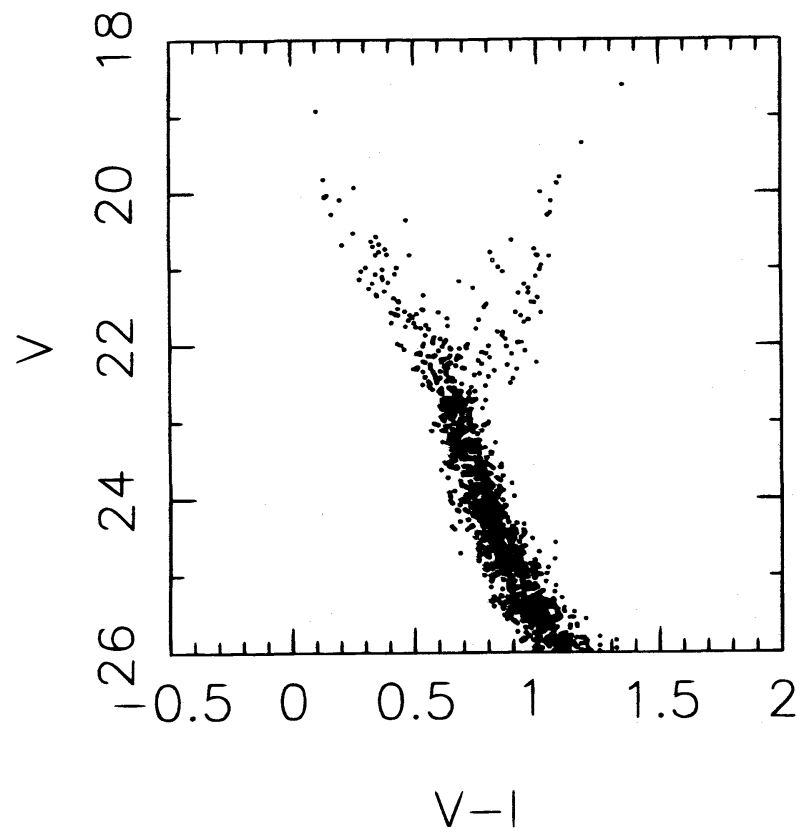

FIG. $3 a$

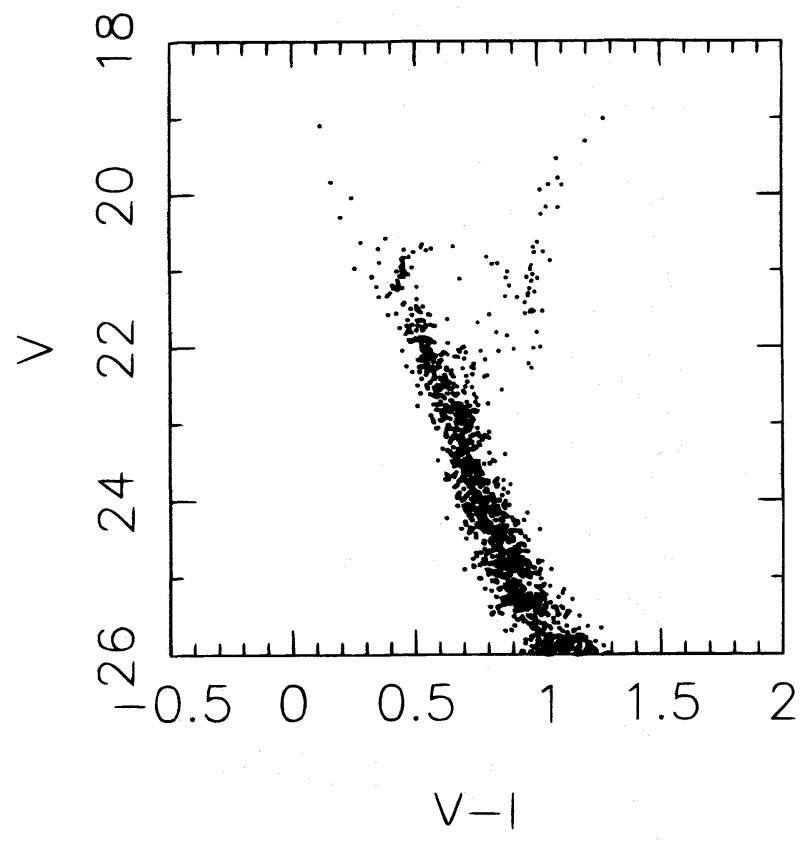

Fig. $3 b$

Fig. 3. - Two illustrative models have been constructed for a CMD containing 1770 stars from the Yale isochrones. The models are designed to fit the morphology but not the details of our observed CMD. The first model (a) assumes a constant SFR (0.5-10 Gyr), while the second (b) shows the impact of adding a short-duration starburst $2 \mathrm{Gyr}$ ago to the constant SFR model. The second model produces a main-sequence turnoff near $V=21.5$, similar to what is observed.

and their main-sequence evolution takes place nearly parallel to the ZAMS (see, e.g., Iben \& Rood 1970). As a result, the width of the main-sequence band is no longer a useful diagnostic of the star formation history, and this technique does not work for stars with ages of more than 3-5 Gyr.

In a real galaxy, the observed main sequence will be additionally broadened by a variety of factors, including measurement errors, variations in distance along the line of sight (a 12\% effect in the LMC if stars are spread over $3 \mathrm{kpc}$, for example), a range in stellar metallicities, and the presence of binary stars. Further uncertainties in matching observations to the stellar models stem from the limitations of model stellar evolution tracks. For example, model stellar lifetimes depend on the choice of opacity tables and the degree of core convective overshoot mixing, which can increase main-sequence lifetimes.

Fortunately, in the LMC disk, the range of metallicities for younger stars is thought to be moderately small. Observations of star clusters and planetary nebulae give mean metallicities extending from about one-half of solar for extreme Population I stars to about $1 / 10$ of solar for the old disk (Da Costa 1991; Dopita et al. 1996). However, the oldest LMC globular star clusters have metallicities of approximately $1 / 100$ of solar (see, e.g., Olszewski 1995), and we must allow for the presence of an old, metal-poor LMC disk component, which produces the LMC disk field RR Lyrae stars (Walker 1993). This span in metallicities will produce moderate variations in the width and location of the main-sequence band (see $\S$ 5.1). Correlations between stellar ages and metallicities can strongly influence our results by changing the mapping between observed magnitudes and stellar masses, and thus stellar ages.

The complication introduced by the inevitable presence of binary stars is that the photometric properties of these systems mimic those of more evolved, single stars. For example, a binary containing two ZAMS $1 M_{\odot}$ stars will be 0.75 mag above the ZAMS, where this system would be mistaken for a more evolved but younger single star. As the mass ratio of a zero-age binary is reduced, the amplitude of the effect declines and the color of the combined system becomes redder (Bertelli et al. 1992; Kroupa, Tout, \& Gilmore 1993). Since most binaries have relatively high mass ratios, the photometric signatures of these systems are generally small, typically less than $0.2 \mathrm{mag}$ in luminosity. The effects of binaries in our data are likely to be near the limits of detectability, and we ignore this correction in the remainder of this paper.

Model results also depend on the adopted distance to the LMC. For example, the distance that we have adopted from Crotts et al. (1995) has an estimated error of $\pm 6 \%$, which introduces a $\pm 12 \%$ range in luminosities. This translates into $\pm 30 \%$ variations in ages derived for stars with masses near $1 M_{\odot}$. A closer distance would increase all of our ages and reduce the length of any period when star formation was possibly quiescent in the LMC.

\subsection{Properties of the Observed Main-Sequence Band}

We model the main-sequence band using the Fagatto et al. (1994) stellar evolution tracks for masses of $0.6-1.8 M_{\odot}$ with abundances of $Y=0.25, Z=0.008$, and $Y=0.24$, $Z=0.0004$, which span the expected range of LMC disk star metallicities. These models use OPAL opacities and include core convective overshoot. As a result of the latter feature, the calculated stellar main-sequence lifetimes are longer than for classical mixing models. This increases lifetimes by less than $10 \%$ for stars which live longer than 4 Gyr and by about $25 \%$ for stars which live for 1 Gyr on the main sequence. The model effective temperatures and luminosities were converted to $V-I$ colors and $M_{V}$ using synthetic $V-I$ colors and bolometric corrections calculated by 
Bessell (1995) from model atmospheres supplied by Kurucz $(1991,1992)$. The resulting model evolutionary tracks are shown plotted over the observations in Figure 4.

No single-metallicity model stellar population fits all of the data. Thus, while the $Z=0.008$ models span the mainsequence band for $M_{V}<4$, these models are too red for lower luminosity stars. An inverse behavior occurs for $Z=0.0004$ models, which provide a reasonable fit to the lower main-sequence band in our data. This combination of features could be understood if LMC disk stars have a bimodal metallicity distribution like that seen for LMC star clusters (Da Costa 1991). In this case, the younger main sequence would have about one-half of solar metallicity and the longer lived, low-mass stars would also include a major component of extremely metal poor stars. The analysis of abundances in planetary nebulae by Dopita et al. (1996) suggests this simple picture is too extreme and thus sets a useful limit for models.

Alternatively, a problem could exist with some aspect of the model tracks, e.g., with the interior structures or atmospheres. The possibility that this offset is due solely to WFPC2 calibration difficulties seems unlikely; the size of the offset is larger than the probable systematic errors (see also Holtzman et al. 1995a). Furthermore, a similar effect is well established in ground-based photometry of LMC star clusters (see, e.g., Mould et al. 1989; Corsi et al. 1994), although the problem is somewhat reduced if a short distance modulus of 18.4 is adopted for the LMC.

Our choice of a longer LMC distance also will tend to minimize derived stellar ages, while our use of convective overshoot models pushes us toward slightly longer evolutionary timescales. Because of these factors, the relative ages derived from the stellar population model fits generally are more accurate than the absolute ages. Clearly these diffi- culties in fitting models to observed CMDs must be sorted out before we can fully understand the evolutionary history of the LMC (see Tolstoy \& Saha 1996). Even so, progress can be made through an examination of the main-sequence band because this type of theoretical comparison primarily depends on the width rather than absolute color of the main-sequence band.

\section{CONSTRAINTS ON THE LMC STAR FORMATION HISTORY}

The observed distribution of stars across lines of constant $M_{V}$ in the main-sequence band are compared with the prediction of a simple stellar population evolutionary model in Figure 5. We use an initial luminosity bin width of $0.4 \mathrm{mag}$ and thereafter $0.2 \mathrm{mag}$ to minimize effects due to the color slope of the main sequence while also maximizing the numbers of stars in each sample. The model results are not sensitive to the form of the initial mass function, and for simplicity we assume a Salpeter IMF and a constant SFR. In this stellar population model, the density of stars along each stellar mass isochrone in a CMD is simply proportional to the lifetime at that position. As expected, the stars in this model cluster near the ZAMS along the blue edge of the main-sequence band for $1<M_{V}<4$, and the distribution of stars redward across the main-sequence band in this luminosity interval depends on the star formation history (see Fig. 4).

\subsection{Constant Star Formation in the Recent Epoch}

Figure 5 shows that constant SFR models provide reasonable fits throughout most of the $1.4<M_{V}<4.2$ mainsequence region. An acceptable fit can be defined as agreement between the model and observed histogram to
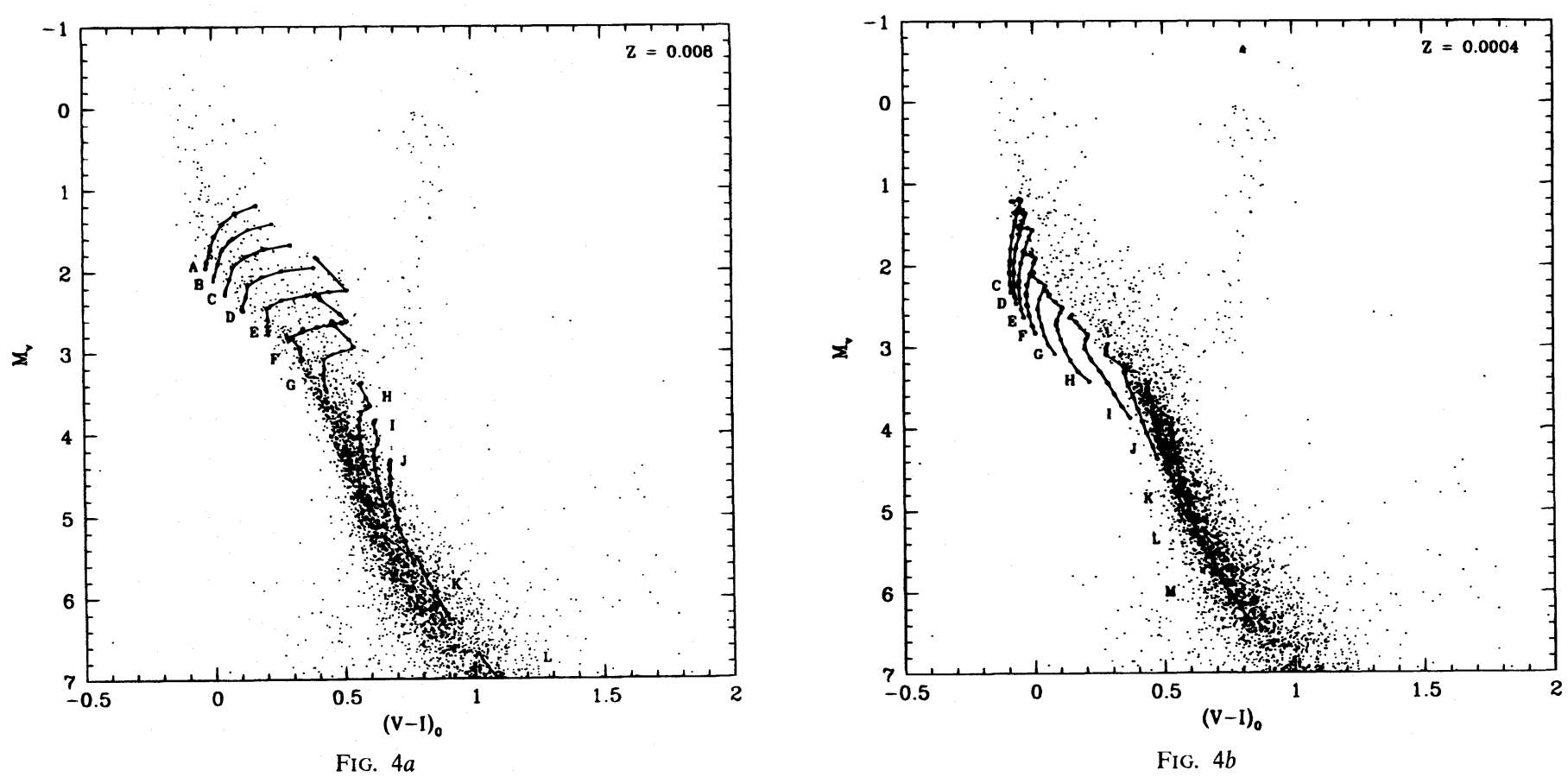

Fig. 4. - Overplots of Faggato et al. (1994) model stellar evolution tracks on the data from Fig. 2. The tracks extend only over $95 \%$ of the calculated main-sequence lifetimes to avoid confusion with short-lived phases, and the points shown are those tabulated by Faggato et al. Model masses are coded by letters extending from $A=1.9 M_{\odot}$ in increments of $0.1 M_{\odot}$ to the lowest mass model $M=0.7 M_{\odot}$. The $1<M_{V}<4$ main sequence is closely fitted by the $Z=0.008$ models, while the observed lower main sequence is too blue and requires a lower metallicity for a proper fit with these models. 

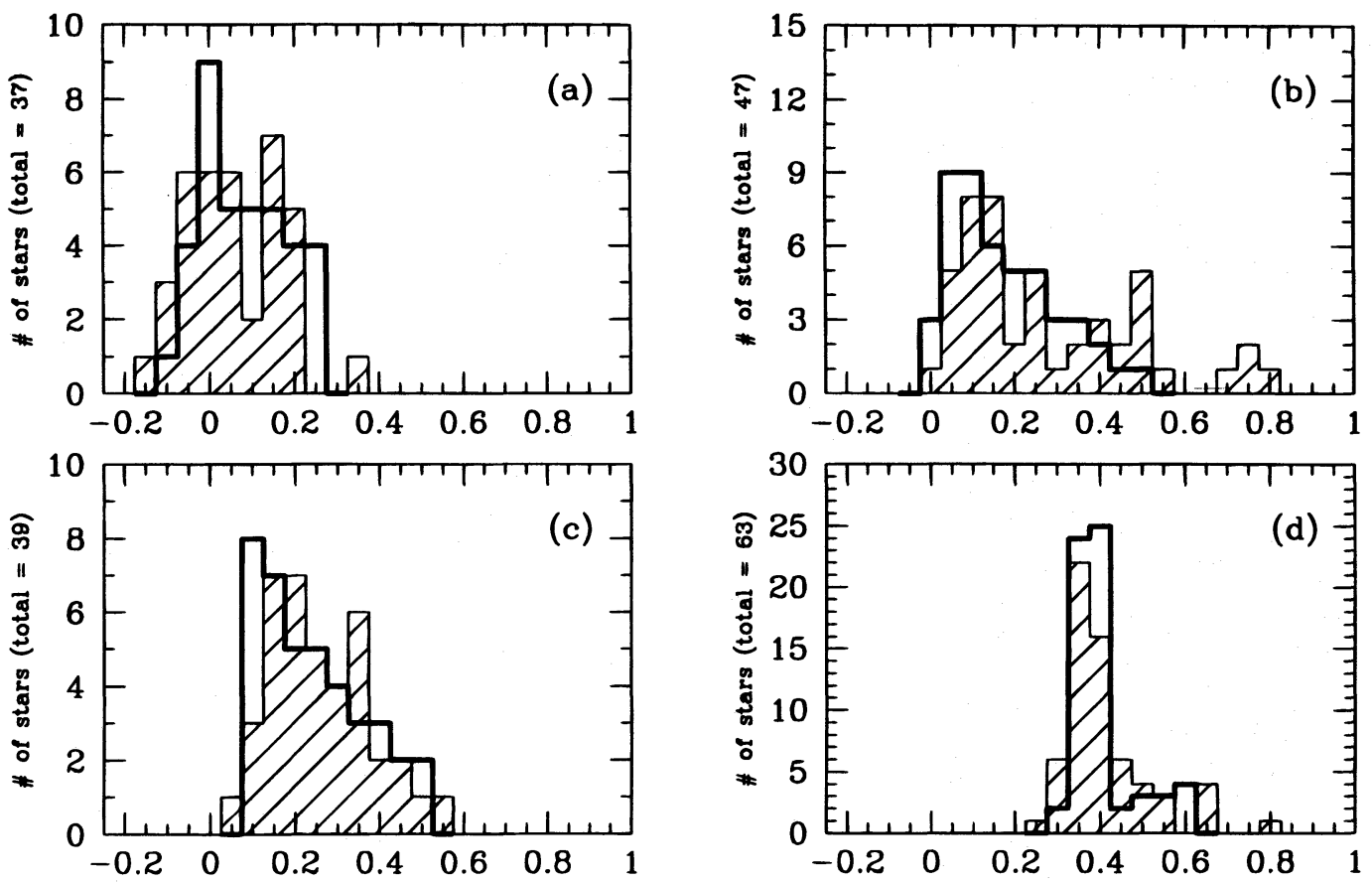

Model Z $=0.008$

(a) $1.4<\mathrm{M}_{\mathrm{v}}<1.8$

(b) $2.0<M_{v}<2.2$

(c) $2.4<\mathrm{M}_{\mathrm{v}}<2.6$

(d) $3.0<M_{v}<3.2$

(e) $4.0<M_{v}<4.2$

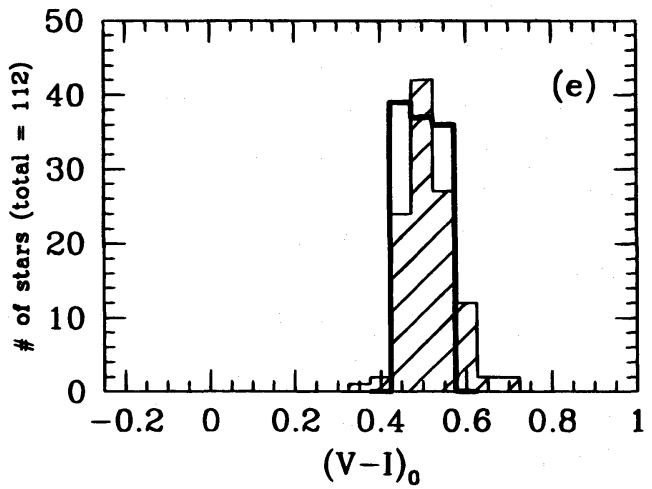

FIG. 5.-Five histograms show the observed (hatched) and predicted (heavy line) numbers of stars from cuts across the CMD at five values of $M_{Y}$. The model predictions assume a constant SFR and provide an acceptable fit to the observations in four of the magnitude intervals. However, the $M_{V}=2.1$ bin shows a red excess and a clump of subgiants that are signatures of the $2 \mathrm{Gyr}$ star formation event, while the narrowness of the main-sequence color spread at $M_{V}=4.1$ argues against a large population of old, extremely metal poor stars.

within the square root of the number of stars in each histogram bin. In particular, the medians of the model and observed distributions agree in all cases except for the $M_{V}=2.1$ slice, which we discuss below. However, because of the small numbers of stars in each sample, SFR fluctuations of $50 \%$ are readily allowed by the statistical uncertainties among the more luminous stars.

The faintest region in which we clearly measure a mainsequence color width is at $M_{V}=3.1$, where the $Z=0.008$ models predict that stars will spend $\approx 1.5-2.3 \mathrm{Gyr}$ of their $2.8 \mathrm{Gyr}$ main-sequence lifetimes in a narrow, near-ZAMS color band. The agreement between the models and observations at this absolute magnitude indicates that the SFR has been roughly constant for most of the past $3 \mathrm{Gyr}$ in the outer LMC disk. During $1 \mathrm{Gyr}$ a star with a peculiar velocity of $1 \mathrm{~km} \mathrm{~s}^{-1}$ travels $1 \mathrm{kpc}$ from its birthplace. Most peculiar velocities are larger than this, and therefore stars with ages of about $1 \mathrm{Gyr}$ or more are widely mixed in the disk of the LMC. A possible exception to this picture of general mixing could apply to stars in the LMC bar, the orbits of which may trap them in the bar over long timescales (see Sparke \& Sellwood 1987). Our field is more than
$3 \mathrm{kpc}$ from the LMC bar and is a good location to sample the outer disk without contamination from the bar.

The agreement between the constant SFR model and observations is not good for stars with luminosities near $M_{V}=2$. On the red side of the main-sequence band, stars have ages of about $1-1.5 \mathrm{Gyr}$, while the blue band is made up of stars with ages of $0-1$ Gyr. The blue side of the observed main-sequence band follows the prediction of the constant SFR model. This implies that the average stellar birthrates during the past $\sim 1 \mathrm{Gyr}$ have been constant to within $50 \%$. An excess of 10 redder, older stars, including the detached clump of subgiants near $(V-I)_{0}=0.75$, is a 3 $\sigma$ deviation from the predictions of our constant SFR model. Evidently, an unusual event took place about $2 \mathrm{Gyr}$ ago, which we see as an excess of red stars in this luminosity zone, and we discuss this event in $\S 5.2$.

Our data become statistically unreliable for mainsequence stars with $M_{V} \leq 1.4$, corresponding to mainsequence ages of about 1 Gyr. The sparse WFPC2 data for brighter main-sequence stars could be consistent with overlapping, multiple turnoffs at higher luminosities, but the numbers of stars are small. In addition, spatial mixing 
within the LMC disk becomes ineffective for stars that are younger than about $1 \mathrm{Gyr}$, and we must be careful not to confuse the naturally discrete nature of the star formation process within a small region as being the signature of an LMC-wide starburst.

The lowest luminosity interval in Figure 5 is slightly below that of the last main-sequence turnoff. In this region, the main-sequence band is centered at $(V-I)_{0}=0.55$ and has a narrow intrinsic color full width at half-maximum of $\Delta(V-I)_{\mathrm{fwhm}}=0.2$. This is fitted in terms of width and central color by the $Z=0.0004$ models, where stars with masses $0.8-0.9 M_{\odot}$ and ages of 4-13 Gyr contribute to the main-sequence band. The $Z=0.008$ models also yield a narrow main-sequence band that is offset by $0.1 \mathrm{mag}$ to the red (see Fig. 4). The main-sequence band width is also $\approx 0.1$ mag or this model, which contains stars with ages of 3-7 Gyr.

The small observed $\Delta(V-I)_{\text {fwhm }}$ color width of the mainsequence band at this luminosity then constrains the metallicity mix of the LMC stellar population in this region. For example, if half of the stars had $Z=0.0004$ and half had $Z=0.008$, we would predict an observed main-sequence color width $\Delta(V-I)_{\mathrm{fwhm}} \approx 0.3$, which is wider than what we measure by a small amount. We therefore conclude that a large population of metal-poor LMC subdwarfs is unlikely to be present in this field, although final confirmation of this view must await deep HST imaging in blue filters. In this respect, the LMC disk is likely to resemble the solar neighborhood of the Milky Way disk, where recent results derived from Hipparchos astrometry indicate that subdwarfs are rare (Perryman et al. 1995).

The luminosity bin at $M_{V}=4$ also shows a statistically significant excess of red stars. This could be produced by a range in stellar metallicities, main-sequence turnoffs of the oldest stars, or binaries. Thus, this feature is not a clear signature of enhanced star formation in the LMC disk during the 3-13 Gyr age interval.

Our data also are a point of contact with observations of the Galactic lower main sequence. The Bessell \& Stringfellow (1993) linear fit to $M_{V}$ and $(V-I)_{0}$ derived from Monet et al. (1992) for a Galactic disk parallax sample falls on the blue edge of our observed main-sequence band at $M_{V}=5$ but is $0.1 \mathrm{mag}$ redder in color than our observations at $M_{V}=6.5$. A major difference between our LMC CMD and that of the local Galactic disk is seen in the location of the subgiant branch associated with the least luminous main-sequence turnoff. The superb Perryman et al. (1995) CMD for the local Galactic disk shows a broad, well-populated subgiant branch extending down to a luminosity of $M_{V} \approx 4$, or about 0.5 mag fainter than what we observe in the LMC.

A similar comparison with a metal-poor galaxy having $[\mathrm{Fe} / \mathrm{H}]=-1.5$ is provided by the Carina dwarf spheroidal galaxy (see, e.g., Gallagher \& Wyse 1994). Smecker-Hane et al. (1996) find that the lowest luminosity Carina subgiants appear above the main sequence at about $M_{V}=3.5-3.7$, or slightly fainter than what we have observed in this LMC field. Smecker-Hane et al. associate these subgiants with a stellar component that is 11-13 Gyr old. An old outer LMC disk component is most readily accommodated if we assume that the old LMC disk stars have metallicities of about $1 \%$ of solar, and for an age of $>10 \mathrm{Gyr}$ we would also prefer a shorter LMC distance modulus. These comparisons therefore suggest that the outer disk of the LMC formed somewhat later than the Milky Way disk in the solar neighborhood.

Assuming that most of the stars in our field are not extremely metal poor, we can place a rough lower bound on the ages of stars in the last main-sequence turnoff. For the Faggato et al. (1994) models and our LMC distance modulus, the oldest turnoff has an age of 6-7 Gyr, depending on the precise metallicity of the oldest stars. For a shorter LMC distance modulus of $(m-M)_{0}=18.4$, the age increases to 8-9 Gyr for the convective overshoot models. Thus, our results agree with those of Bertelli et al. (1992), Westerlund et al. (1995), Vallenari et al. (1996a, b), and earlier studies in suggesting that the disk of the LMC is considerably younger than the oldest LMC globular clusters (see also reviews by Westerlund 1990; Feast 1995; Olszewski 1995).

\subsection{The 2 Gyr Star Formation Event}

A subgiant branch is seen in our data near $M_{V}=2$. The detection of even a few stars of this luminosity in short-lived evolutionary phases within the Hertzsprung gap is surprising and requires a high initial birthrate. Subgiants in the gap evolve very rapidly, and stars in the Fagotto et al. (1994) models spend about $1 / 20$ of their main-sequence lifetime in this phase. The Fagotto et al. model closest to this region is for a $1.4 M_{\odot}$ star for $Z=0.008$. Its main-sequence lifetime is $2.28 \mathrm{Gyr}$. We see 10 stars distributed across the gap within a $\approx 0.2 \mathrm{mag}$ luminosity interval around $M_{V}=$ 2.1. The narrow range in observed luminosities implies that the subgiants have a small range in masses and were born during a $\approx 0.1 \mathrm{Gyr}$ time interval.

In a constant SFR model we would then require a population of on the order of 200 main-sequence stars to produce the observed number of subgiants. Currently, only about 50 stars are within the $M_{V}=3$ region of the mainsequence band which will eventually feed into the observed subgiant branch. We therefore predict a maximum of three subgiants for a constant SFR model. To obtain the observed number of subgiants, the SFR in this region about 2 Gyr ago must have been a factor of 3 or more higher for at least $0.1 \mathrm{Gyr}$ than the mean rate observed now. However, it is unlikely that the SFR could have increased by as much as a factor of 10 during $0.1 \mathrm{Gyr}$. This size of a SFR fluctuation would produce a pronounced step in the luminosity function, which is not seen.

A spatially localized factor of 3-10 SFR increase during 0.1 Gyr could be produced by the normal star formation process if we are seeing the results of a large star formation complex which made stars in a diffuse cloud (e.g., LMC Constellation III; Nail \& Shapley 1953; see also Efremov 1979). On the other hand, during 2 Gyr, stars will diffuse over $>2 \mathrm{kpc}$ from their birthplaces in the LMC disk. The few subgiants that we observe in this region therefore could be signposts of a large-scale star-forming event in the disk of the LMC.

A search for star clusters in this sector of the LMC with ages near $2 \mathrm{Gyr}$ (i.e., subgiant branches near $M_{V}=2.1$ ) would be useful as a means to define the extent of the $2 \mathrm{Gyr}$ star-forming event. Evidence for a global starburst would be revealed by enhanced SFRs in a significant portion of the LMC during the same time period. While the ages of rich star clusters distributed throughout the LMC establish that star formation was unusually active between 2 and $4 \mathrm{Gyr}$ ago, the cluster ages are not sufficiently accurate to see if the 
same short-duration, galaxy-wide starburst produced the subgiants that we see here, as well as a large number of star clusters (see, e.g., Mould \& Aaronson 1982; Hodge 1989; van den Bergh 1991; Girardi et al. 1995).

However, the bulk of the outer disk LMC stars born within the past $\approx 3$ Gyr evidently were not formed in a few high-amplitude bursts. If this had been the case, we would expect to see several well-defined main-sequence turnoffs, whereas we actually see that most turnoffs have blended together. The only good evidence for a starburst is the 2 Gyr old subgiant branch, and this involves a maximum of $25 \%$ of the stars born in the past $\approx 2$ Gyr. This result is in apparent contradiction with indications of a rapid increase in metallicity in the LMC beginning about 2-3 Gyr ago (Dopita et al. 1996), which imply a very large scale starforming event (see also Girardi et al. 1995). We do not yet have a complete picture of this key phase in the evolution of the LMC, but it does seem that something extraordinary occurred in the LMC 2-3 Gyr ago.

The 2 Gyr subgiants are relatively isolated in the CMD. We do not find an increasing population of fainter subgiants below this sequence. This implies that following the 2 Gyr, event, the SFR declined. Information about the longer term evolution of the LMC disk is contained in the region of our CMD around $M_{V}=3.2$ where the last mainsequence turnoff is found. This part of the CMD is complicated to interpret because stellar ages and metallicities can play against each other in such a way that stars with factor of 2 ranges in ages can have similar colors and luminosities. We conclude that the SFR was at or below the average levels of the past Gyr in the LMC during roughly 3-6 Gyr in the past (see also Vallenari et al. 1996a).

\section{DISCUSSION AND SUMMARY}

Deep WFPC2 observations of a patch of the LMC outer disk provide the first deep $V-I$ color-magnitude diagram for the LMC outer disk stellar population. In this initial analysis, we have emphasized modeling the width of the main-sequence band, which is well resolved in color along the $1<M_{V}<4$ region of the lower main sequence and is populated by stars with ages of about 1-3 Gyr. We have also analyzed the subgiant branches as a way to detect times when the SFR was enhanced, and by mapping mainsequence turnoffs, we extend our knowledge of the LMC outer disk star formation history back to ages of at least 6 Gyr.

These models indicate that SFRs have remained roughly constant over most of the past $\approx 3$ Gyr. However, a major SFR increase occurred about $2 \mathrm{Gyr}$ ago based on $Z=0.008$, core convective overshoot stellar evolution models (Faggato et al. 1994). This event could have produced a significant fraction $(\approx 25 \%)$ of the stellar population formed in the past 2 Gyr in our field, is likely to have included much of the LMC disk, and could qualify as a starburst. Observations of additional LMC fields are required to test this hypothesis fully, but supporting evidence for this burst exists in cluster age distributions and abundance patterns (see, e.g., Girardi et al. 1995; Dopita et al. 1996). The LMC bar may act as an independent dynamical subsystem over long timescales, and therefore its evolutionary history also must be considered to obtain a global star formation history of the LMC.

Since the LMC is a satellite of the Milky Way, it is subject to episodic tidal perturbations during perigalacti- con, which may be enhanced by LMC-SMC interactions. It is therefore tempting to ascribe a bursty star formation history in the LMC to the effects of these interactions (Westerlund 1990). However, the precise orbits of the Magellanic Clouds are not yet known, although interactions with the Milky Way every few Gyr are a feature of currently preferred models (Lin, Jones, \& Klemola 1995). A simple tidally induced star formation model for the LMC also suggests that the minimum SFR should have occurred during the last apogalacticon, or about $1.5 \mathrm{Gyr}$ ago. We do not see strong evidence for this SFR minimum. Better dynamical models and star formation history time lines are needed to test the tidally modulated SFR model for the LMC.

The interpretation of a star formation history from a CMD for a single field is both complicated and aided by the many paths that stars may take from their formation sites to the locations where they are observed. For younger stars, the mixing-length scale is short; e.g., mixing over kiloparsec scales requires $0.1-1$ Gyr. Stars on near-circular galactic orbits also generally require several orbital periods to mix fully in angle around an annulus. When we include the 0.1-1 Gyr diffusion timescale for stars to escape from star clusters, which are numerous in the LMC (Hodge 1980), we see that our field sample should become a fair sample of the outer LMC disk for timescales of $\geq 1 \mathrm{Gyr}$.

Our best model for recent the production of stars in the LMC disk is then one of moderately variable but essentially continuous star formation during the past 1-3 Gyr, punctuated by a major event $\approx 2 \mathrm{Gyr}$ ago. Before this event, the SFR evidently did not experience any significant peaks in the preceding several Gyr, a result that is consistent with the evidence from planetary nebulae for a doubling of metal abundances about 2-3 Gyr ago (Dopita et al. 1996). We therefore have yet to find compelling evidence for major starbursts in the LMC 3-6 Gyr ago (Feast 1995).

The star formation history that we derive for the LMC disk is in basic agreement with other studies of the star formation history of the LMC in which SFRs are enhanced in relatively recent times (see, e.g., Bertelli et al. 1992; Westerlund et al. 1995; Girardi et al. 1995; Vallenari et al. 1996a). The very recent star formation history $(t<0.1 \mathrm{Gyr})$ is not well determined from our observations of such a small region, but the presence of the $0.1 \mathrm{Gyr}$ age, luminous blue star cluster NGC 1866 near our field, and the extension of the main sequence to short-lived stars prove that star formation has been active in this part of the LMC disk during the past 0.1-1 Gyr. Apparently, in recent times, the LMC disk has behaved as we would expect based on observations of global SFRs in nearby Magellanic irregular galaxies: the recent SFR fluctuates but large excursions from the mean are comparatively rare.

The long-term star formation history of the LMC disk, however, is a remarkable astrophysical puzzle. Both the metallicity and the star formation history appear to have rather discontinuous histories. While the oldest LMC globular clusters are metal poor, their formation evidently was followed by a period of low star formation rates before the main disk began to form in earnest about 6-8 Gyr ago. During this time, the metallicity evidently rose slowly to near the current value, in seeming contradiction to the lack of star formation (see, e.g., Da Costa 1991; Dopita et al. 1996). The continuing exploration of the age-metallicity relationship for stars in the various components of the 
LMC will provide us with an important means for understanding how galaxies evolve.

We thank Paul Hodge, Tammy Smecker-Hane, Linda Sparke, and Nick Suntzeff for discussions and comments on this project; Andrew Cole for his assistance in preparing this paper; and Rosemary Wyse and an anonymous referee for comments which have improved this manuscript. We also thank Mike Bessell and Mike Dopita for providing results in advance of publication. This work has been carried out as part of the WFPC2 Investigation Team GTO scientific research program and was supported by NASA contract NAS7-1260 to the Jet Propulsion Laboratory.

\section{REFERENCES}

Bertelli, G., Mateo, M., Chiosi, C., \& Bressan, A. 1992, ApJ, 388,400

Bessell, M. S. 1995, private communication

Bessell, M. S., \& Stringfellow, G. S. 1993, ARA\&A, 31, 433

Broadhurst, T. J., Ellis, R. S., \& Shanks, T. 1988, MNRAS, 235, 827

Burrows, C. J., ed. 1995, Wide Field and Planetary Camera 2 Handbook (Baltimore: Space Telescope Science Institute)

Butcher, H. 1977, ApJ, 216, 327

Corsi, C. E., Buonanno, R., Fusi Pecci, F., Ferraro, F. R., Testa, V., \& Greggio, L. 1994, MNRAS, 271, 385

Cowie, L. L., Songaila, A., \& Hu, E. M. 1991, Nature, 354, 460

Crotts, A. P. S., Kunkel, W. E., \& Heathcote, S. R. 1995, ApJ, 438, 724

Da Costa, G. S. 1991, in IAU Symp. 148, The Magellanic Clouds, ed. R. Haynes \& D. Milne (Dordrecht: Kluwer), 183

Dopita, M., et al. 1996, preprint

Efremov, Yu. N. 1979, Soviet Astron. Lett., 6,12

Eggleton, P., Fitchett, M. J., \& Tout, C. A. 1989, ApJ, 347,998

Fagatto, F., Bressan, A. Bertelli, G., \& Chiosi, C. 1994, A\&AS, 105, 29

Feast, M. J. 1995, in IAU Symp. 164, Stellar Populations, ed. P. C. van der Kruit \& G. Gilmore (Dordecht: Kluwer), 153

Frogel, J. A., \& Blanco, V. M. 1983, ApJ, 274, L57

Gallagher, J. S., Hunter, D. A., \& Tutukov, A. V. 1984, ApJ, 284, 544

Gallagher, J. S., \& Wyse, R. F. G. 1994, PASP, 106, 1225

Girardi, L. Chiosi, C. Bertelli, G. \& Bressan, A. 1995, A\&A, 298,87

Green, E. M., Demarque, P., \& King, C. R. 1987, The Revised Yale Isochrones and Luminosity Functions (New Haven: Yale Univ. Obs.)

Hardy, E., Buano, R., Corsi, C. E., Janes, K. A., \& Schommer, R. A. 1984, ApJ, 278, 592

Herschel, J. F. W. 1847, Results of Astronomical Observations Made During the Years 1834, 5, 6, 7, 8 at the Cape of Good Hope (London: Smith, Elder)

Hodge, P. 1980, AJ, 85, 423

$$
\begin{aligned}
& \text { 1987, PASP, 99, } 730 \\
& \text { 1988, PASP, 100, } 576
\end{aligned}
$$

1989, ARA\&A, 27,139

Holtzman, J. A., Burrows, C. J., Casertano, S., Hester, J. J., Trauger, J. T., Watson, A. M., \& Worthy, G. M. 1995a, PASP, 107,1065

Holtzman, J. A., Hester, J. J., Casertano, S., Trauger, J. T. Watson, A. M. $\&$ the WFPC2 Investigation Definition Team. 1995b, PASP, 107, 156

Holtzman, J. A., et al. 1996, in preparation

Iben, I., Jr., \& Rood, R. 1970, ApJ, 159, 605

Jensen, J., Mould, J., \& Reid, N. 1988, ApJS, 67,77

Koo, D. C., Guzmán, R., Faber, S. M., Illingworth, G. D., Bershady, M. A., Kron, R. G., \& Takamiya, M. 1995, ApJ, 440,L49
Kroupa, P., Tout, C. A., \& Gilmore, G. 1993, MNRAS, 262,545

Kurucz, R. L. 1991, in IAU Symp. 149, The Stellar Populations of Galaxies, ed. B. Barbuy \& A. Renzini (Dordrecht: Kluwer), 225

1992, private communication

Lilly, S. 1993, ApJ, 411, 501

Lin, D. N. C., Jones, B. F. \& Klemola, A. R. 1995, ApJ, 439, 652

Mighell, K. J., \& Butcher, H. R. 1992, A\&A, 255, 26

Monet, D. G., Dahn, C. C., Vrba, F. J., Harris, H. C., Pier, J. R., Luginbuhl, C. B., \& Ables, H. D. 1992 , AJ, 103, 638

Mould, J., \& Aaronson, M. 1982, ApJ, 263, 629

Mould, J. R., Kristian, J., Nemec, J., Aaronson, M., \& Jensen, J. 1989, ApJ, 339,84

Nail, V. M. \& Shapley, H. 1953, Proc. Natl. Acad. Sci.,39, 358

O'Connell, R. W., Gallagher, J. S., \& Hunter, D. A. 1994, ApJ, 433, 65

Olszewski, E. W. 1995, in IAU Symp. 164, Stellar Populations, ed. P. C. van der Kruit \& G. Gilmore (Dordecht: Kluwer), 181

Perryman, M. A. C., et al. 1995, A\&A, 304,69

Renzini, A., \& Fusi-Pecci, F. 1988, ARA\&A, 26,199

Reid, I. N., Hughes, S. M. G., \& Glass, I. S. 1995, MNRAS, 275, 331

Scalo, J. M. 1986, Fundam. Cosmic Phys., 11, 1

Smecker-Hane, T. A., Stetson, P. B., Hesser, J. E., \& Lehnert, M. D. 1994, AJ, 108, 507

Smecker-Hane, T. A. Stetson, P. B. Hesser, J. E. \& VandenBerg, D. A 1996, in ASP Conf. Ser., From Stars to Galaxies: The Impact of Stellar Physics on Galaxy Evolution, ed. C. Leitherer, U. Fritze-von Alvensleben, \& J. Huchra (San Francisco: ASP), in press

Soderblom, R. D., Duncan, D. K., \& Johnson, D. R. H. 1991, ApJ, 375, 722

Sparke, L. S., \& Sellwood, J. A. 1987, MNRAS, 225, 653

Stryker, L. 1983, ApJ, 266, 82

. 1984, ApJS, 55, 127

Suntzeff, N. B., Schommer, R. A., Olszewski, E. W., \& Walker, A. W. 1992, AJ, 104, 1743

Tolstoy, E., \& Saha, A. 1996, ApJ, 462, 672

Trauger, J. T., et al. 1994, ApJ, 435, L3

Vallenari, A., Chiosi, C., Bertelli, G., Aparicio, A., \& Ortolani, S. 1996a, preprint

Vallenari, A., Chiosi, C., Bertelli, G., \& Ortolani, S. 1996b, preprint van den Bergh, S. 1991, ApJ, 369,

Walker, A. R. 1993, in IAU Colloq. 139, New Perspectives on Stellar Pulsation and Pulsating Variable Stars, ed. J. M. Nemec \& J. M. Matthews (Cambridge: Cambridge Univ. Press), 15

Westerlund, B. E. 1990, A\&A Rev., 2,29

Westerlund, B. E., Linde, P., \& Lyngå, G. 1995, A\&A, 298, 39 